\title{
Inverse Analysis from a CONDORCET Robustness Denotation of Valued Outranking Relations
}

\author{
Raymond Bisdorff ${ }^{1}$, Patrick Meyer ${ }^{2,3}$, and Thomas Veneziano ${ }^{1}$ \\ ${ }^{1}$ University of Luxembourg \\ Faculty of Sciences, Technology, and Communication \\ Computer Science and Communications Research Unit \\ Interdisciplinary Lab for Intelligent and Adaptive Systems \\ 6, rue Richard Coudenhove-Kalergi, L-1359 Luxembourg \\ \{raymond.bisdorff, thomas.veneziano\}@uni.lu \\ ${ }^{2}$ Institut Télécom, Télécom Bretagne \\ UMR CNRS 3192 Lab-STICC \\ Technopôle Brest Iroise CS 83818 F-29238 Brest Cedex 3, France \\ patrick.meyer@telecom-bretagne.eu \\ ${ }^{3}$ Université européenne de Bretagne
}

\begin{abstract}
In this article we develop an indirect approach for assessing criteria significance weights from the robustness of the significance that a decision maker acknowledges for his pairwise outranking statements in a Multiple Criteria Decision Aiding process. The main result consists in showing that with the help of a mixed integer linear programming model this kind of a priori knowledge is sufficient for estimating adequate numerical significance weights.
\end{abstract}

Keywords: inverse Multiple Criteria Decision Analysis, significance weights ellicitation, uncertainty, robustness.

\section{Introduction}

We consider a decision situation in which a finite set of decision alternatives is evaluated on a finite family of performance criteria. A decision maker is willing to pairwisely compare these alternatives according to the outranking paradigm. One considers indeed that an alternative $a$ outranks an alternative $b$ when a significant majority of criteria validates the fact that $a$ is performing at least as good as $b$ and there is no criterion where $b$ seriously outperforms $a$ [1]. To assess when such a significant majority of criteria validates an outranking situation requires a more or less precise numerical knowledge of the significance of each criterion in the multiple criteria preference aggregation. Two different approaches exist to specify theses values:

- either via direct preference information, where the criteria significance is first assessed and then the aggregated outranking situations are computed,

- or, via indirect preference information, where some a priori partial knowledge of the resulting aggregated outranking is used in order to infer plausible estimators of the criteria significance. 
In this article we exclusively concentrate on the indirect preference information approach. Similar approaches, mostly in the domain of Multiple Attribute Value Theory, already appeared in the literature where they are generally called disaggregation/aggregation or ordinal regression methods [2/3/4/5/6|7/8]. In analogy with corresponding techniques in inferential statistics, we prefer to group all indirect preference information modeling techniques under the generic term inverse Multiple Criteria Decision Analysis. The innovative a priori knowledge on which we focus our inverse analysis here is the robustness of the significant majorities that the decision maker acknowledges for his pairwise comparisons with respect to all potential significance weights, a fact we call the CONDORCET robustness of the outranking situation in the sequel of this article. The main result of our article is to show that this kind of a priori knowledge alone is sufficient for estimating numerical significance weights.

The article is organised as follows: in the next section, we define the CONDORCET robustness denotation of valued outranking relations and then, in Section 3 we briefly detail the way of computing it. Afterwards, in Section 4 we present a mathematical model for estimating the significance weights followed by some brief remarks on practical application issues.

\section{Defining the Condorcet Robustness Denotation of Valued Outranking Relations}

Let $A=\{x, y, z, \ldots\}$ be a finite set of $n>1$ potential decision alternatives and $F=\left\{g_{1}, \ldots, g_{m}\right\}$ a coherent finite family of $m>1$ criteria.

The alternatives are evaluated on each criterion on real performance scales to which an indifference $q_{i}$ and a preference $p_{i}$ discrimination threshold (for all $g_{i}$ in $F$ ) is associated [1. The performance of alternative $x$ on criterion $g_{i}$ is denoted $x_{i}$.

In order to characterise a local at least as good as situation 910 between any two alternatives $x$ and $y$ of $A$, with each criterion $g_{i}$ is associated a double threshold order $S_{i}$ whose numerical representation is given by:

$$
S_{i}(x, y)=\left\{\begin{aligned}
1 & \text { if } x_{i}+q_{i} \geqslant y_{i}, \\
0 & \text { if } x_{i}+p_{i} \leqslant y_{i} \\
0.5 & \text { otherwise. }
\end{aligned}\right.
$$

Furthermore, we associate with each criterion $g_{i} \in F$ a rational significance weight $w_{i}$ which represents the contribution of $g_{i}$ to the overall warrant or not of the at least as good as preference situation between all pairs of alternatives. Let $W=\left\{w_{i}: g_{i} \in F\right\}$ be the set of relative significance weights associated with $F$ such that $0<w_{i}<1\left(\forall g_{i} \in F\right)$ and $\sum_{g_{i} \in F} w_{i}=1$ and let $\mathcal{W}$ be the set of such significance weights sets.

The overall valued outranking relation, denoted $\widetilde{S}^{W}$, aggregating the partial at least as good as situations, is given by :

$$
\widetilde{S}^{W}(x, y)=\sum_{w_{i} \in W} w_{i} \cdot S_{i}(x, y), \forall(x, y) \in A \times A .
$$


$\widetilde{S}^{W}(x, y)$ is thus evaluated in the rational interval $[0,1]$ with the following semantics [9]:

- $\widetilde{S}^{W}(x, y)=1$ indicates that all criteria warrant unanimously the "at least as good as" preference situation between $x$ and $y$;

- $S^{W}(x, y)>0.5$ indicates that a majority of criteria warrant the "at least as good as" preference situation between $x$ and $y$;

- $S^{W}(x, y)=0.5$ indicates a balanced situation where the criteria warranting the "at least as good as" preference situation between $x$ and $y$ are exactly as significant as those who do not warrant this situation;

- $\widetilde{S}^{W}(x, y)<0.5$ indicates that a majority of criteria do not warrant the "at least as good as" preference situation between $x$ and $y$;

- $\widetilde{S}^{W}(x, y)=0$ indicates that all criteria warrant unanimously the negation of the "at least as good as" preference situation between $x$ and $y$.

Let $\succsim_{W}$ be the preorde1 1 on $F$ associated with the natural $\geqslant$ relation on the set of significance weights $W . \sim_{W}$ induces $r$ ordered equivalence classes $\Pi_{1}^{W} \succ_{W}$ $\ldots \succ_{W} \Pi_{r}^{W}(1 \leq r \leq m)$. The criteria of an equivalence class have the same significance weight in $W$ and for $i<j$, those of $\Pi_{i}^{W}$ have a higher significance weight than those of $\Pi_{j}^{W}$. Let $\mathcal{W}_{\succsim_{W}} \subset \mathcal{W}$ denote the set of all significance weights sets that are preorder-compatible with $\succsim_{W}$.

Let $W \in \mathcal{W}$. The CONDORCET robustness denotation $2\left[12\right.$ of $\widetilde{S}^{W}$, denoted $\llbracket \widetilde{S}^{W} \rrbracket$, is defined, for all $(x, y) \in A \times A$, as follows:

$\llbracket \widetilde{S}^{W} \rrbracket(x, y)=\left\{\begin{aligned} 3 & \text { if } \widetilde{S}^{V}(x, y)=1 \forall V \in \mathcal{W} ; \\ 2 & \text { if }\left[\widetilde{S}^{V}(x, y)>0.5 \forall V \in \mathcal{W}_{\succsim W}\right] \wedge\left[\exists V^{\prime} \in \mathcal{W}: \widetilde{S}^{V^{\prime}}(x, y)<1\right] ; \\ 1 & \text { if }\left[\widetilde{S}^{W}(x, y)>0.5\right] \wedge\left[\exists V^{\prime} \in \mathcal{W}_{\succsim W}: \widetilde{S}^{V^{\prime}}(x, y) \leqslant 0.5\right] ; \\ 0 & \text { if } \widetilde{S}^{W}(x, y)=0.5 ; \\ -1 & \text { if }\left[\widetilde{S}^{W}(x, y)<0.5\right] \wedge\left[\exists V^{\prime} \in \mathcal{W}_{\succsim W}: \widetilde{S}^{V^{\prime}}(x, y) \geqslant 0.5\right] ; \\ -2 & \text { if }\left[\widetilde{S}^{V}(x, y)<0.5 \forall V \in \mathcal{W}_{{ }^{\prime}}\right] \wedge\left[\exists V^{\prime} \in \mathcal{W}: \widetilde{S}^{V^{\prime}}(x, y)>0\right] ; \\ -3 & \text { if } \widetilde{S}^{V}(x, y)=0 \forall V \in \mathcal{W} ;\end{aligned}\right.$

with the following semantics:

- $\llbracket \widetilde{S}^{W} \rrbracket(x, y)= \pm 3$ if all criteria unanimously warrant (resp. do not warrant) the outranking situation between $x$ and $y$;

- $\llbracket \widetilde{S}^{W} \rrbracket(x, y)= \pm 2$ if a significant majority of criteria warrants (resp. does not warrant) the outranking situation between $x$ and $y$ for all $\succsim_{W}$-compatible weights sets;

- $\llbracket \widetilde{S}^{W} \rrbracket(x, y)= \pm 1$ if a significant majority of criteria warrants (respectively does not warrant) this outranking situation for $W$ but not for all $\succsim_{W^{-}}$ compatible weights sets;

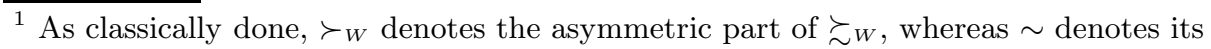
symmetric part.

${ }^{2}$ The simple majority validated outranking relation $S^{W}(x, y)$ such that $\widetilde{S}^{W}(x, y)>0.5$ is generally called the CONDORCET relation (see Barbut [11]), in honours of the Marquis DE CONDORCET (1743-1794) who first promoted social choice procedures based on pairwise simple majority votings. 
- $\llbracket \widetilde{S}^{W} \rrbracket(x, y)=0$ if the total significance of the warranting criteria is exactly balanced by the total significance of the not warranting criteria for $W$.

The careful reader may have noticed that, in the presence of veto thresholds as defined in [10, if a veto situation occurs in the comparison of a couple of alternatives, the associated CONDORCET robustness denotation is -3 , as the overall outranking relation $\widetilde{S}^{W}$ equals 0 , disregarding the criteria significance weights.

\section{Computing the Condorcet Robustness Denotation}

In this section, we briefly explain how to obtain the CONDORCET robustness denotation. Further details can be found in [12].

Let us consider the following numerical example to illustrate our purpose throughout this paper.

Example. Consider a set $A=\{a, b, c, d, e\}$ of five decision alternatives and a consistent family $F$ of three cardinal criteria $\left\{g_{1}, g_{2}, g_{4}\right\}$ measuring performances on rational scales from 0.0 to 100.0 and two ordinal criteria $\left\{g_{3}, g_{5}\right\}$ measuring performances on a discrete ordinal scale from 0 to 10 . Criterion $g_{2}$ is a cost-type criterion on which performances have to be minimised, whereas the four other are benefit-type criteria, i.e. the higher the performance is the better a decision alternative is considered.

Table 1 presents the randomly generated performances of the alternatives on each criterion. Notice the significance weights set $W$ shown in the third column which induces the significance ordering $\left\{g_{1}\right\} \succ_{W}\left\{g_{4}\right\} \succ_{W}\left\{g_{3}\right\} \succ_{W}\left\{g_{5}\right\} \succ_{W}$ $\left\{g_{2}\right\}$.

Let us start by presenting the notation which allows us to detail the construction of the CONDORCET robustness denotation associated with a valued outranking relation $\widetilde{S}^{W}$ and a significance weights set $W$.

Let $c_{k}^{W}(x, y)$ be the sum of "at least as good as" characteristics $S_{i}(x, y)$ for all criteria $g_{i} \in \Pi_{k}^{W}$, and $\overline{c_{k}^{W}}(x, y)$ the sum of the negation $\left(1.0-S_{i}(x, y)\right)$ of these characteristics. Furthermore, let $C_{k}^{W}(x, y)=\sum_{i=1}^{k} c_{i}^{W}(x, y)$ be the cumulative sum of "at least as good as" characteristics for all criteria having significance at

Table 1. Performance table

\begin{tabular}{|c|c|c|c|c|c|c|c|c|c|}
\hline $\begin{array}{l}\text { crit. } \\
(F)\end{array}$ & $\begin{array}{l}\text { pref. } \\
\text { dir. }\end{array}$ & $\begin{array}{l}\text { weights } \\
(W)\end{array}$ & $\begin{array}{c}\text { deci } \\
a\end{array}$ & $\begin{array}{c}\text { ision } a \\
b\end{array}$ & $\begin{array}{l}\text { altern } \\
\quad c\end{array}$ & native & $\begin{array}{l}\text { es }(A) \\
e\end{array}$ & $\begin{array}{l}\text { thres } \\
\text { indiff }\end{array}$ & $\begin{array}{l}\text { lolds } \\
\text { pref. }\end{array}$ \\
\hline$g_{1}$ & $\max$ & $5 / 11$ & 70.9 & 61.8 & 90.2 & 31.2 & 33.1 & 5.0 & 8.0 \\
\hline$g_{2}$ & $\min$ & $1 / 15$ & 20.9 & 17.1 & 76.3 & 69.2 & 35.5 & 3.0 & 6.0 \\
\hline$g_{3}$ & $\max$ & $3 / 15$ & 1 & 4 & 6 & 8 & 6 & 0 & 1 \\
\hline$g_{4}$ & $\max$ & $4 / 15$ & 17.3 & 46.3 & 24.5 & 40.6 & 68.2 & 6.0 & 7.0 \\
\hline$g_{5}$ & $\max$ & $2 / 15$ & 2 & 1 & 8 & 2 & 6 & 0 & 1 \\
\hline
\end{tabular}


least equal to the one associated to $\Pi_{k}^{W}$, and let $\overline{C_{k}^{W}}(x, y)=\sum_{i=1}^{k} \overline{c_{i}^{W}}(x, y)$ be the cumulative sum of the negation of these characteristics, for all $k$ in $\{1, \ldots, r\}$.

In the absence of \pm 3 denotations, the following proposition gives us a test for the presence of a \pm 2 denotation:

\section{Proposition 1 (Bisdorff [12])}

$$
\llbracket \widetilde{S}^{W} \rrbracket(x, y)=2 \Longleftrightarrow\left\{\begin{array}{l}
\forall k \in 1, \ldots, r: C_{k}^{W}(x, y) \geqslant \overline{C_{k}^{W}}(x, y) ; \\
\exists k \in 1, \ldots, r: C_{k}^{W}(x, y)>\overline{C_{k}^{W}}(x, y) .
\end{array}\right.
$$

The negative -2 denotation corresponds to similar conditions with reversed inequalities.

The \pm 2 denotation test of Proposition 1 corresponds in fact to the verification of stochastic dominance-like conditions (see 12]).

$\mathrm{A} \pm 1$ CondorCET robustness denotation, corresponding to the observation of a weighted majority (resp. minority) in the absence of the \pm 2 case, is simply verified as follows:

$$
\llbracket \widetilde{S}^{W} \rrbracket(x, y)= \pm 1 \Longleftrightarrow\left(\left(\widetilde{S}^{W}(x, y) \gtrless 0.5\right) \wedge \llbracket \widetilde{S}^{W} \rrbracket(x, y) \neq \pm 2\right)
$$

Example. Back to the example, we can now compute the CONDORCET robustness denotation associated with the outranking relation. Let us detail these calculations for the following two couples, $(b, c)$ and $(a, d)$. Recall that the significance ordering is given by a five-class preorder $\left\{g_{1}\right\} \succ_{W}\left\{g_{4}\right\} \succ_{W}\left\{g_{3}\right\} \succ_{W}\left\{g_{5}\right\} \succ_{W}\left\{g_{2}\right\}$.

We can easily verify via Table 2 that $\llbracket \widetilde{S}^{W} \rrbracket(b, c)=-2$. Besides we can see that $\llbracket \widetilde{S}^{W} \rrbracket(a, d) \neq \pm 2$. Since $\tilde{S}^{W}(a, d)=0.53>0.5$, we finally have $\llbracket \widetilde{S}^{W} \rrbracket(a, d)=1$. Table 3 presents the outranking relation $\widetilde{S}^{W}$ and its corresponding CondorCET robustness denotation $\llbracket \widetilde{S}^{W} \rrbracket$ for all pairs of alternatives of $A \times A$.

The issue we address in this paper is now the following. Consider that we have given a performance table as shown in Table 1, but without any explicit significance weights information, as well as a CONDORCET robustness denotation $\llbracket \widetilde{S}^{W} \rrbracket$ similar to the one shown in the right part of Table 3, with $W$ unknown. Is it possible to infer from these information alone the apparent significance weights of the criteria? In other words, may we compute on the basis of the given information a preorder $\succsim$ on the criteria and a numerical instance $W^{*}$ of a $\succsim$-compatible

Table 2. Cumulative sums for couples $(b, c)$ and $(a, d)$

\begin{tabular}{l|l|l|l|l||l|l|l|l}
\hline \multicolumn{1}{c}{||$c_{i}^{W}(b, c) \mid \overline{c_{i}^{W}}(b, c)$} & $C_{i}^{W}(b, c) \mid \overline{C_{i}^{W}}(b, c)$ & $\left|c_{i}^{W}(a, d)\right| \overline{c_{i}^{W}}(a, d)\left|C_{i}^{W}(a, d)\right| \overline{C_{i}^{W}}(a, d)$ \\
\hline$\Pi_{1}^{W}$ & 0 & 1 & 0 & $\mathbf{1}$ & 1 & 0 & $\mathbf{1}$ & 0 \\
$\Pi_{2}^{W}$ & 1 & 0 & $\mathbf{1}$ & $\mathbf{1}$ & 0 & 1 & $\mathbf{1}$ & $\mathbf{1}$ \\
$\Pi_{3}^{W}$ & 0 & 1 & 1 & $\mathbf{2}$ & 0 & 1 & 1 & $\mathbf{2}$ \\
$\Pi_{4}^{W}$ & 0 & 1 & 1 & $\mathbf{3}$ & 1 & 0 & $\mathbf{2}$ & $\mathbf{2}$ \\
$\Pi_{5}^{W}$ & 1 & 0 & 2 & $\mathbf{3}$ & 1 & 0 & $\mathbf{3}$ & 2 \\
\hline
\end{tabular}


Table 3. Global outranking with CONDORCET robustness denotation

\begin{tabular}{c|ccccc|ccccc}
\hline & \multicolumn{5}{|c|}{$\widetilde{S}^{W}$} & \multicolumn{5}{|c}{$\llbracket \widetilde{S}^{W} \rrbracket$} \\
$A$ & $a$ & $b$ & $c$ & $d$ & $e$ & $a$ & $b$ & $c$ & $d$ & $e$ \\
\hline$a$ & - & .50 & .07 & .53 & .40 & - & 0 & -2 & 1 & -1 \\
$b$ & .53 & - & .33 & .67 & .40 & 1 & - & -2 & 2 & -1 \\
$c$ & .93 & .67 & - & .47 & .67 & 2 & 2 & - & -1 & 2 \\
$d$ & .60 & .60 & .53 & - & .53 & 1 & 1 & 1 & - & 1 \\
$e$ & .60 & .60 & .53 & .80 & - & 1 & 1 & 1 & 2 & - \\
\hline
\end{tabular}

weights set which satisfies the given CONDORCET robustness denotation $\llbracket \widetilde{S}^{W} \rrbracket$, i.e. $W^{*}$ and $\succsim$ are such that $\llbracket \widetilde{S}^{W^{*}} \rrbracket=\llbracket \widetilde{S}^{W} \rrbracket$ ?

\section{Inferring the Criteria Significance Weights}

To solve this estimation problem we are going to formulate a mixed integer linear programming model.

We start with characterising a constraint model for every possible CoNDORCET robustness denotation except the \pm 3 ones. Indeed, we may ignore unanimous positive and negative $( \pm 3)$ robustness denotations as they concern in fact the trivial pairwise comparison of alternatives that are either Pareto dominating or Pareto dominated. Their aggregated outranking situation is thus always unanimously warranted (resp. not warranted), independently of any particular criteria significance weights. These denotations therefore do not contain any specific information for inferring the particular significance of an individual criterion.

We denote $A_{ \pm 2}^{2}$ (resp. $A_{ \pm 1}^{2}$ or $\left.A_{0}^{2}\right)$ the set of pairs $(x, y)$ of alternatives such that $\llbracket \widetilde{S}^{W} \rrbracket(x, y)= \pm 2($ resp. \pm 1 or 0$)$.

As the criterion significance weights are supposed to be rational, we may without loss of generality restrict our estimation problem to integer weight sets. Thus every criterion may get an integer significance weight $w_{i} \in[1, M]$, where $M$ denotes the maximal admissible value. Limiting our purpose to genuine decision aid situations, we may choose this bound in practical applications to be equal to the number $m$ of criteria.

We denote $P_{m \times M}$ a Boolean $(0,1)$-matrix, with general term $\left[p_{i, u}\right]$, that characterises row-wise the number of weight units allocated to criterion $g_{i}$. Formally, the row $i$ represents the decomposition of the weight associated to $g_{i}$ into $M$ bits in a unary base (little-endian) and thus $\sum_{u=1}^{M} p_{i, u}=w_{i}$.

The fact that every criterion $g_{i}$ of $F$ must have a strictly positive significance may thus be expressed with the help of the following constraint:

$$
\sum_{g_{i} \in F} p_{i, 1}=m
$$


At least one weight unit is allocated to every criterion, i.e. $p_{i, 1}=1$ for all $g_{i} \in F$. As an example, if $g_{i}$ has an integer weight of 3 and if we decide that $M=5$, then the $i$ th row of $P_{m \times 5}$ is given by $(1,1,1,0,0)$.

The required cumulative semantics of $P_{m \times M}$ is therefore achieved with the following set of constraints:

$$
p_{i, u} \geqslant p_{i, u+1} \quad(\forall i=1, \ldots, m, \forall u=1, \ldots, M-1) .
$$

\subsection{Constraints for $\llbracket \widetilde{S}^{W} \rrbracket(x, y)= \pm 2$ Conditions}

Let us now translate Proposition 1 to a computable set of constraints.

\section{Corollary 1}

When considering integer weights, Proposition 1 may be reformulated as:

$$
\llbracket \widetilde{S}^{W} \rrbracket(x, y)=2 \Longleftrightarrow\left\{\begin{array}{l}
\forall u \in 1, \ldots, \max w_{i}: C_{u}^{\prime W}(x, y) \geqslant \overline{C_{u}^{\prime W}}(x, y) \\
\exists u \in 1, \ldots, \max w_{i}: C_{u}^{\prime W}(x, y)>\overline{C_{u}^{\prime W}}(x, y)
\end{array}\right.
$$

where $C_{u}^{\prime W}(x, y)\left(\operatorname{resp} . \overline{C_{u}^{\prime W}}(x, y)\right)$ is the sum of all $S_{i}(x, y)$ (resp. $\left.1-S_{i}(x, y)\right)$ such that the significance weight $w_{i} \leq u$. The negative -2 denotation corresponds again to similar conditions with reversed inequalities.

Proof. We easily verify that all constraints from Proposition 1 are present in the corollary (for the set $U=\left\{u / \exists w_{i} \in W, w_{i}=u\right\}$ of indexes). For all other values of $u$ the constraints are redundant.

This leads to the property that $p_{i, u}=1 \Longleftrightarrow w_{i} \geq u$ and we directly obtain:

$$
C_{u}^{\prime W}(x, y)=\sum_{g_{i} \in F}\left(p_{i, u} \cdot S_{i}(x, y)\right) .
$$

In order to model now the $\llbracket \widetilde{S}^{W} \rrbracket(x, y)= \pm 2$ conditions, we introduce for all pairs $(x, y) \in A_{+2}^{2}$ the following set of constraints:

$$
\sum_{g_{i} \in F}\left(p_{i, u} \cdot\left[S_{i}(x, y)-\overline{S_{i}}(x, y)\right]\right) \geqslant b_{u}(x, y) \quad(\forall u=1, \ldots, M),
$$

where $\overline{S_{i}}$ is the negation $\left(1-S_{i}\right)$ of the criterion's double threshold order characteristic function, and where the $b_{u}(x, y)$ are Boolean $(0,1)$ variables for each pair of alternatives and each equi-significance level $u$ in $\{1, \ldots, M\}$. Note that the negative -2 denotation again corresponds to a similar inequation with a reversed inequality and negative $b_{u}(x, y)$. These binary variables allow us to impose at least one case of strict inequality for each $(x, y) \in A_{ \pm 2}^{2}$ as required in Corollary 1 via the following constraints:

$$
\sum_{u=1}^{m} b_{u}(x, y) \geqslant 1, \quad\left(\forall(x, y) \in A_{ \pm 2}^{2}\right)
$$




\subsection{Constraints for $\llbracket \widetilde{S}^{W} \rrbracket(x, y)= \pm 1$ Conditions}

In order to introduce the $\llbracket \widetilde{S}^{W} \rrbracket(x, y)= \pm 1$ conditions, we may formulate for all pairs $(x, y) \in A_{ \pm 1}^{2}$ the following set of constraints:

$$
\sum_{g_{i} \in F}\left(\sum_{u=1}^{M} p_{i, u}\right) \cdot \pm\left(S_{i}(x, y)-\bar{S}_{i}(x, y)\right) \geqslant 1 \forall(x, y) \in A_{ \pm 1}^{2},
$$

where the factor $\left(\sum_{u=1}^{M} p_{i, u}\right)$ represents the integer value of the estimated weight $w_{i}$ of criterion $g_{i}$.

Recall that a CONDORCET robustness denotation of \pm 2 represents an outranking situation which is validated (or non-validated) for all possible weights sets compatible with the given significance preorder. Such a situation therefore represents a robust validation by the decision maker, and should as such be considered highly trustful. Consequently, if the decision maker imposes a \pm 1 or 0 CONDORCET robustness, this can be considered as more anecdotical. In practical situations, it might happen that the CONDORCET robustness given by the decision maker might not be compatible with the underlying problem. To avoid not finding any solution, we relax Constraints (11) by adding positive slack variables which have to be minimised in order to satisfy best possibly the constraints:

$$
\sum_{g_{i} \in F}\left(\sum_{u=1}^{M} p_{i, u}\right) \cdot \pm\left(S_{i}(x, y)-\bar{S}_{i}(x, y)\right) \pm s^{ \pm 1}(x, y) \geqslant 1 \forall(x, y) \in A_{ \pm 1}^{2} .
$$

\subsection{Constraints for $\llbracket \widetilde{S}^{w} \rrbracket(x, y)=0$ Conditions}

Similarly as in the previous section, for all pairs $(x, y) \in A_{0}^{2}$, we formulate the corresponding set of soft equality constraints:

$$
\sum_{g_{i} \in F}\left(\sum_{u=1}^{M} p_{i, u}\right) \cdot\left(S_{i}(x, y)-\bar{S}_{i}(x, y)\right)+s_{+}^{0}(x, y)-s_{-}^{0}(x, y)=0 .
$$

\subsection{Objective Function}

Finally, our overall objective is to determine a significance weights set $W^{*}$ which:

- satisfies all the $\llbracket \widetilde{S}^{W^{*}} \rrbracket(x, y)= \pm 2$ constraints,

- respects the $\llbracket \widetilde{S}^{W^{*}} \rrbracket(x, y)= \pm 1$ and $\llbracket \widetilde{S}^{W^{*}} \rrbracket(x, y)=0$ constraints as well as possible, and

- gives the smallest possible weights $w_{i}\left(g_{i} \in F\right)$ (which, in practice, tends to use the least possible number of equi-significance classes).

Therefore, we introduce the following objective function which is to be minimised: 


$$
\begin{aligned}
& K_{1}\left(\sum_{g_{i} \in F} \sum_{u=1}^{M} p_{i, u}\right) \\
- & K_{2}\left(\sum_{u=1}^{M}\left(\sum_{(x, y) \in A_{ \pm 2}^{2}} b_{u}(x, y)\right)\right) \\
+ & K_{3}\left(\sum_{(x, y) \in A_{ \pm 1}^{2}} s^{ \pm 1}(x, y)\right)+K_{4}\left(\sum_{(x, y) \in A_{0}^{2}}\left(s_{+}^{0}(x, y)+s_{-}^{0}(x, y)\right)\right)
\end{aligned}
$$

where $K_{1} \ldots K_{4}$ are parametric constants used for the correct hierarchical ordering of the four sub-goals. Note that (3) is not necessary for solving our problem, but it guarantees the strictest possible enforcing of the $\llbracket \widetilde{S}^{W} \rrbracket(x, y)= \pm 2$ constraints with strict inequalities.

In summary, we obtain the following linear mixed integer program which covers all positive, negative and zero CONDORCET robustness denotations:

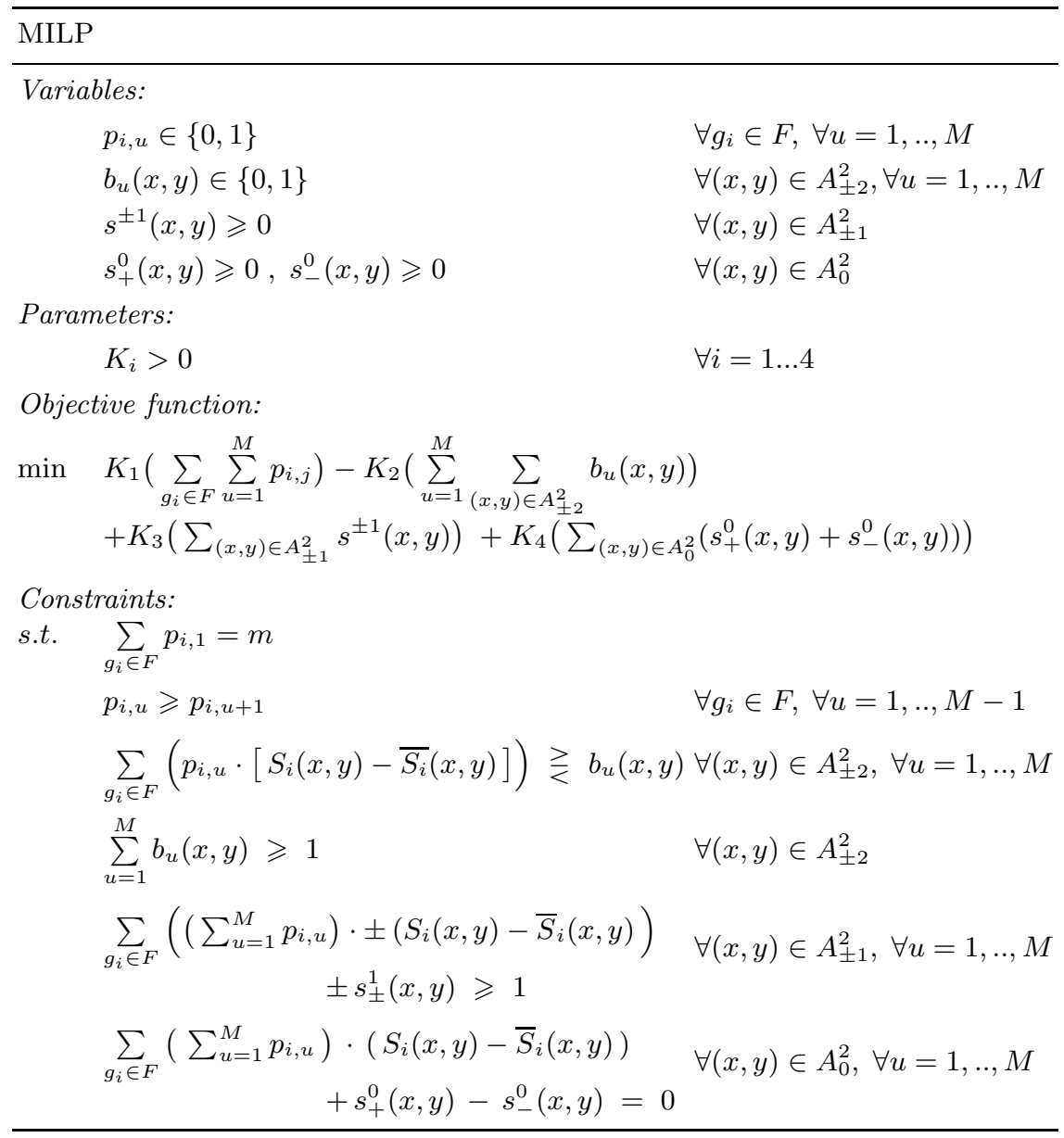


Table 5. Optimal MILP solution for our example with estimated significance weights

\begin{tabular}{|c|c|c|c|}
\hline & 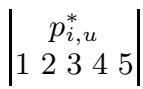 & $W^{*}$ & $W$ \\
\hline & & $5 / 13$ & \\
\hline & & $1 / 13$ & $1 / 1$ \\
\hline & & $3 / 13$ & $3 / 1$ \\
\hline & 00 & $3 / 13$ & $4 / 1$ \\
\hline & $\begin{array}{lllll}1 & 0 & 0 & 0 & 0\end{array}$ & $1 / 13$ & $2 / 1$ \\
\hline
\end{tabular}

Let $P^{*}=\left[p_{i, u}^{*}\right]$ be an optimal solution of the MILP model. We may calculate the estimated significance weights as the row sum of $\left[p_{i, u}^{*}\right]$, i.e. $w_{i}^{*}=\sum_{u=1}^{M} p_{i, u}^{*}$ for all criteria $g_{i} \in F$ and thus recover the corresponding significance preorder $\succsim_{W^{*}}$.

Example. Let us reconsider our example. Solving MILP with Cplex 11.0 gives the optimal $P^{*}$ matrix shown in Table 國. The resulting estimated normalised weights are: $w_{1}^{*}=0.385, w_{2}^{*}=0.077, w_{3}^{*}=0.231, w_{4}^{*}=0.231$ and $w_{5}^{*}=0.077$, whereas the real weights that we initially generated are : $w_{1}=0.333, w_{2}=0.067, w_{3}=0.200$, $w_{4}=0.267$ and $w_{5}=0.133$.

All constraints related to the 6 pairs $(x, y) \in A_{ \pm 2}^{2}$ are positively verified, as well as those concerning the 13 pairs $(x, y) \in A_{ \pm 1}^{2}$ and the pair $(a, b) \in A_{0}^{2}$. Therefore we get with $K_{i}=1 \forall i=1 \ldots 4$ the optimal value of -22 for the objective function $(13-7 \cdot 5+0+0)$.

The original linear significance order: $\left\{g_{1}\right\} \succ_{W}\left\{g_{4}\right\} \succ_{W}\left\{g_{3}\right\} \succ_{W}\left\{g_{5}\right\} \succ_{W}\left\{g_{2}\right\}$ is reconstructed as a three-level significance preorder: $\left\{g_{1}\right\} \succ_{W^{*}}\left\{g_{3}, g_{4}\right\} \succ_{W^{*}}\left\{g_{2}, g_{5}\right\}$. Recomputing the corresponding overall outranking relation we obtain the estimated $\widetilde{S}^{W^{*}}$ relation (see Table 6 ), which admits an identical CONDORCET robustness denotation as the original $\widetilde{S}^{W}$ relation.

The example that we detailed through this article illustrates the fact that the reconstruction from the CONDORCET robustness denotation alone of the significance weights set following the original valued outranking relation is in general

Table 6. Global outranking relation with inferred significance weights

\begin{tabular}{|c|c|c|c|c|c|}
\hline \multirow{2}{*}{$A$} & estimated $\widetilde{S}^{W^{*}}$ & \multicolumn{4}{|c|}{ original $\widetilde{S}^{W}$} \\
\hline & $\begin{array}{lllll}x_{1} & x_{2} & x_{3} & x_{4} & x_{5}\end{array}$ & $x_{1} x_{2}$ & & & $x_{5}$ \\
\hline$x_{1}$ & .50 .08 .54 .46 & $\mid-.50$ & .07 & .53 & .40 \\
\hline$x_{2}$ & $.54-.31 .69 .46$ & $.53-$ & .33 & .67 & .40 \\
\hline$x_{3}$ & $.92 .69-.46 .69$ & .93 .67 & - & & .67 \\
\hline$x_{4}$ & $.54 .54 .54-.62$ & .53 .53 & .53 & - & .53 \\
\hline$x_{5}$ & $.54 .54 .54 .77 \quad-$ & .53 .53 & .53 & .80 & - \\
\hline
\end{tabular}

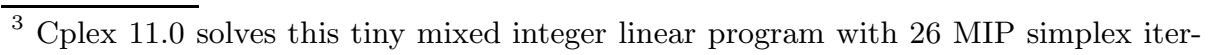
ations, 0 branch-and-bound nodes and 4 Gomory cuts.
} 
not unique and not completely faithful. Several admissible significance preorders and numerical weights sets might indeed support the same robustness denotation and some tuning of the MILP objective function may be necessary depending on the decision aid goal we intend to follow in order to get a useful result.

\subsection{Practical Application Issues}

If we apply the MILP model with Cplex 11.0, associated with an AMPL front end modeler on more or less real-sized random multiple criteria decision problems (20 alternatives evaluated on 13 criteria) we observe quite reasonable solving times on an 6 threaded standard application server. Depending on the maximal value $M$ allowed for an individual criterion significance weight we indeed obtain average computation times of 2.5 seconds for $M=7$ up to 2 minutes for $M=13$.

As already mentioned, for a given value of $M$, the MILP might have some non zero slacks. In such a case, as our purpose is here to find a solution without any slacks, we need to increase the value of $M$ to reduce the slacks. In practice, we simply reiterate the resolution, with $M$ slightly incremented. Notice that 1 more unit for $M$ produces $m$ new binary variables (from $p_{1, M+1}$ to $p_{m, M+1}$ ), increasing significantly the computation time as we have noticed before.

Furthermore, a great number of problems may be solved using values $M$ much lower than the number of criteria such that the number of columns of matrix $P$ is generally overestimated. Consequently, to limit the expected computation time, we recommend to set the initial maximal admissible value $M$ to the requested depth of the estimated significance preorder and to increase it only if necessary.

\section{Conclusion}

In this paper we have presented an innovative method to determine significance weights of criteria in Multiple Criteria Decision Aid, while guaranteeing a high degree of robustness and therefore a high reliability of the outranking relation.

In the future we plan to examine the questioning of a decision maker in order to obtain valuable information for the determination of the significance weights from robustness affirmations. This involves the analysis of the decision maker's responses as well as the study of the interactive use of the algorithm presented in this paper. In particular we intend to restrict the decision maker's intervention on a few pairs of alternatives and infer the outranking relation for those remaining.

\section{References}

1. Roy, B., Bouyssou, D.: Aide Multicritère à la Décision: Méthodes et Cas. Economica, Paris (1993)

2. Jacquet-Lagrèze, E., Siskos, Y.: Assessing a set of additive utility functions for multicriteria decision making: the UTA method. European Journal of Operational Research 10, 151-164 (1982)

3. Mousseau, V., Słowinski, R.: Inferring an Electre TRI model from assignment examples. Journal of Global Optimization 12(2), 157-174 (1998) 
4. Mousseau, V., Dias, L.C., Figueira, J., Gomes, C., Clímaco, J.N.: Resolving inconsistencies among constraints on the parameters of an MCDA model. European Journal of Operational Research 147(1), 72-93 (2003)

5. Siskos, Y., Grigoroudis, E., Matsatsinis, N.F.: Uta methods. In: Figueira, J., Greco, S., Ehrgott, M. (eds.) Multiple Criteria Decision Analysis: State of the Art Surveys, pp. 297-344. Springer, Boston (2005)

6. Grabisch, M., Kojadinovic, I., Meyer, P.: A review of capacity identification methods for Choquet integral based multi-attribute utility theory: Applications of the Kappalab R package. European Journal of Operational Research 186, 766-785 (2008)

7. Greco, S., Mousseau, V., Słowinski, R.: Ordinal regression revisited: multiple criteria ranking using a set of additive value functions. European Journal of Operational Research 191(2), 415-435 (2008)

8. Meyer, P., Marichal, J.-L., Bisdorff, R.: Disaggregation of bipolar-valued outranking relations. In: An, L.T.H., Bouvry, P., Tao, P.D. (eds.) MCO. Communications in Computer and Information Science, vol. 14, pp. 204-213. Springer, Heidelberg (2008)

9. Bisdorff, R.: Logical foundation of multicriteria preference aggregation. In: Bouyssou, D., et al. (eds.) Aiding Decisions with Multiple Criteria, pp. 379-403. Kluwer Academic Publishers, Dordrecht (2002)

10. Bisdorff, R., Meyer, P., Roubens, M.: Rubis: a bipolar-valued outranking method for the best choice decision problem. 4OR: A Quarterly Journal of Operations Research 6(2), 143-165 (2008)

11. Barbut, M.: Médianes, Condorcet et Kendall. Mathématiques et Sciences Humaines 69, 9-13 (1980)

12. Bisdorff, R.: Concordant outranking with multiple criteria of ordinal significance. 4OR 2(4), 293-308 (2004) 